\title{
Identification in transgenic animals of the Drosophila decapentaplegic sequences required for embryonic dorsal pattern formation
}

\author{
F. Michael Hoffmann and William Goodman \\ McArdle Laboratory for Cancer Research, University of Wisconsin, Madison, Wisconsin 53706 USA
}

\begin{abstract}
Mutant alleles of the Drosophila decapentaplegic (dpp) gene affect embryonic dorsal-ventral pattern formation, larval viability, and adult cuticle formation from the imaginal disks. The $d p p$ DNA required for this array of functions spans almost $50 \mathrm{~kb}$. We report that the embryonic lethal, ventralizing alleles of the $d p p$ gene are rescued in transgenic animals by an 8-kb fragment of the wild-type dpp DNA. Full rescue, from embryonic lethality to adult viability, is obtained in two situations: in animals hemizygous for the haplolethal dpp gene, and in animals hemizygous for either of two recessive embryonic lethal alleles. In embryos null for $d p p$, the transformation of dorsal cuticle to ventral cuticle is blocked by one copy of the $d p p$ transposon; two copies permit the hatching of the larvae. The portion of $d p p$ sufficient for these embryonic functions encodes a protein with homology to the transforming growth factor- $\beta$ (TGF- $\beta$ ) family of proteins (Padgett et al. 1987). The larval and imaginal disk functions of $d p p$ are not rescued by the $8-\mathrm{kb}$ portion of the gene and must require additional sequences from the $50 \mathrm{~kb}$ of DNA.
\end{abstract}

[Key Words: Drosophila; pattern formation; transgenic]

Received April 14, 1987; revised version accepted June 1, 1987.

The Drosophila decapentaplegic (dpp) gene is required for dorsal-ventral pattern formation in the early embryo (Gelbart et al. 1985; Irish 1986), for viability of the larva (Segal and Gelbart 1985), and for the growth of the imaginal disk cells that produce much of the cuticle of the adult fly (Spencer et al. 1982). The gene spans almost 50 $\mathrm{kb}$ of DNA (F.M. Hoffmann, R.D. St. Johnston, and W.M. Gelbart, unpubl.| and is predicted to encode a protein product, on the basis of the DNA sequence, that is homologous to the mammalian regulatory factors transforming growth factor- $\beta$ (TGF- $\beta$ ), inhibin- $\beta$, and Müllerian inhibiting substance (Padgett et al. 1987).

The $d p p$ gene originally was identified by a series of mutant alleles that affect the development of the fly during embryogenesis and at metamorphosis (Spencer et al. 1982). The disk alleles result in the failure to develop properly the adult cuticular structures derived from the imaginal disks. Striking defects in the adult cuticle derived from 15 of the imaginal disks led to the name of the gene. Each allele affecting the imaginal disks exhibits complete penetrance and uniform expressivity; however, a spectrum of phenotypes is seen among the group of disk alleles. Progressively more proximal cuticular structures along the proximal-distal axis of adult appendages are absent in animals with the more severe alleles (Spencer et al. 1982).
The embryonic alleles, a second group of $d p p$ mutant alleles, fail to complement the disk alleles, as evidenced by the absence of some imaginal disk-derived cuticular structures in heterozygotes. These mutant alleles also disrupt embryogenesis. Genetically they are of two types: dominant lethals that can be rescued by a duplication of the $d p p$ gene, called $d p p^{H i n}$ alleles for "haploinsufficient-decapentaplegic" and recessive embryonic lethals, called $d p p^{h i n r}$ and $d p p^{e}$ alleles. In $d p p$-null embryos, in which both copies of the $d p p$ gene are haplolethal alleles, the dorsal cuticle is transformed to a ventral cuticle. The dorsal-to-ventral transformation is characterized by the presence of ventral denticle hairs on lateral and dorsal surfaces, thereby forming rings of denticle hairs around the circumference of the embryo (Gelbart et al. 1985; Irish 1986). Therefore, $d p p$ function is required for proper embryonic development along the dorsal-ventral axis of the embryonic epidermis. A number of other Drosophila genes are also implicated in development along the dorsal-ventral axis of the embryo, including 10 maternal-effect genes in which lossof-function mutations produce dorsalized embryos (Anderson and Nusslein-Volhard 1984). A ventralized phenotype is observed in Toll dominant mutations (Anderson et al. 1985a,b). The $d p p$ gene is epistatic to three of the maternal-effect dorsalizing genes, dorsal. 
pelle, and Toll, so dpp may function on the same developmental pathway "downstream" of these genes (Irish 1986).

A third class of $d p p$ mutations is made up of alleles that fail to complement a mutation originally called shortvein. These $d p p$ alleles also fail to complement the recessive embryonic lethals at $d p p$ and, in some cases, fail to complement $d p p$ disk alleles. By these genetic criteria, shortvein and its alleles were defined as part of the $d p p$ gene (Segal and Gelbart 1985). Several of the $d p p^{s h v}$ alleles exhibit a larval lethal phenotype associated with a slow larval growth rate.

The $d p p$ gene was cloned by a chromosomal walk from an adjacent middle repetitive element (F.M. Hoffmann, R.D. St. Johnston, and W.M. Gelbart, unpubl.). Mapping of chromosomal rearrangements associated with $d p p$ mutant phenotypes indicates that approximately $50 \mathrm{~kb}$ of genomic DNA is part of the $d p p$ gene. Five overlapping transcripts, ranging in size from 3.5 to $5.0 \mathrm{~kb}$, are differentially modulated during development (Gelbart et al. 1985). The transcripts have two common $3^{\prime}$ exons, from the Hin domain, and several different 5' exons, four of which map to the shortvein domain of the gene. None of the transcripts extends into the disk domain. The DNA sequence of cDNA clones reveals an open reading frame encoded entirely on the two 3' exons from the Hin domain. The predicted protein sequence extends over 455 or 588 amino acids, depending on which of two in-frame initiation codons is used. The 100 amino acids at the carboxyl terminus have extensive amino acid sequence homology with a family of mammalian growth factors or regulatory peptides that includes TGF- $\beta$, inhibin- $\beta$, and Müllerian-inhibiting substance (Padgett et al. 1987).

To understand the genetic complexity implicated by the three genetic domains and the molecular complexity implicated by the five transcripts, we are dissecting the gene into the individual segments required for specific functions. Our assay is the restoration of biological function in a $d p p$ mutant animal. We begin our functional dissection of the gene with a piece of DNA spanning the Hin region because of the requirement for this region in all $d p p$ functions.

\section{Results}

Obtaining the dpp transgenic lines

The hypothesis that the DNA between positions 83 and 91 on the DNA map might be sufficient for the role of $d p p$ in embryonic pattern formation was based on the location of $d p p$ mutant alleles on the DNA. $d p p$ mutant lesions were mapped on the DNA by Southern blot analysis of mutant DNAs and by in situ hybridization of cloned $d p p$ DNA to polytene chromosomes bearing $d p p$ mutant chromosomal rearrangements (F.M. Hoffmann, R.D. St. Johnston, and W.M. Gelbart, unpubl.). $d p p^{s h v}$ mutant lesions fell distal to position 83 on the chromosome walk, and imaginal disk-specific $d p p$ mutant lesions fell proximal to position 91 on the DNA map. The genetic data indicated that the region of $d p p$ essential for embryonic development lies between the shortvein and disk regions (Spencer et al. 1982; Segal and Gelbart 1985). Recently, a number of $d p p^{\text {Hin }}$ alleles have been mapped within the 83-91 DNA fragment (Gelbart et al. 1985). Because chromosomal rearrangement breakpoints responsible for shortvein and disk mutant phenotypes map to either side of the 83-91 fragment and have no effect on the embryonic morphology or embryonic viability of the organism, we thought that $d p p$ function residing on the 83-91 fragment might be expressed properly in the embryo even if the 83-91 fragment was not contiguous with the flanking $d p p$ sequences.

To produce transgenic animals for this portion of the $d p p$ gene, we used a plasmid containing sequences essential for P-element transposon-mediated DNA transformation and selection of stable transformants. The plasmid pPA-1 (provided by Dr. James Posakony, University of California, San Diegol has the selectable Drosophila alcohol dehydrogenase ( $a d h)$ gene flanked by the terminal sequences of the P element. The vector DNA was opened at a unique EcoRI site, and an $8-\mathrm{kb} E c o$ RI fragment of $d p p$ DNA, isolated from a $\lambda$ phage clone obtained from the Maniatis-Laurer genomic library, was inserted (Fig. 1). Recombinant clones were isolated with the $d p p$ DNA inserted in each of the two possible orientations relative to the adh gene and P-element promoters (pPAdpp8391 and pPAdpp9183). The 8-kb EcoRI fragment of $d p p$ contains the entire protein-coding sequence identified on cDNA clones (Padgett et al. 1987). The orientation of the open reading frame found on $d p p$ cDNA clones is noted in Figure 1, as are the locations of the two in-frame AUGs and the termination codon as deduced from aligning the cDNA sequence (Padgett et al. 1987) with the genomic sequence (D. Gunn and F.M. Hoffmann, unpubl.). Also included on this portion of $d p p$ DNA is one of the two tRNA ${ }^{\text {Tyr }}$ genes found within the dpp gene (Gelbart et al. 1985).

The standard P-element-mediated DNA transformation strategy was used (Rubin and Spradling 1982). The chromosomal locations of the integrated transposons were mapped genetically by segregation analysis with the dominant markers Curly $(C y)$ on the second chromosome and Ultrabithorax $(U b x)$ on the third chromosome. After establishment of balanced stocks of the transgenic lines, the sites of insertion were determined by in situ hybridization of ${ }^{3} \mathrm{H}$-labeled $d p p$ DNA (fragment 83-91) to polytene chromosomes. The data for the transgenic lines carrying $d p p 8391$ and, in one case, the opposite orientation $d p p 9183$ are shown in Table 1.

\section{Transposon rescue of the Hin lethality}

To assay the transgenic lines for $d p p$ function encoded on the transposon, we mated virgin females to males from stocks of several $d p p^{\text {Hin }}$ alleles. The alleles are haplo-lethals that produce ventralized cuticle; included among these alleles are deletions of the entire gene (Spencer et al. 1982; Irish 1986). For maintenance of stocks of these haplo-lethal alleles, a complete duplication of the $d p p$ gene is present on the $\mathbf{X}$ chromosome. When males from these stocks are mated to females that 


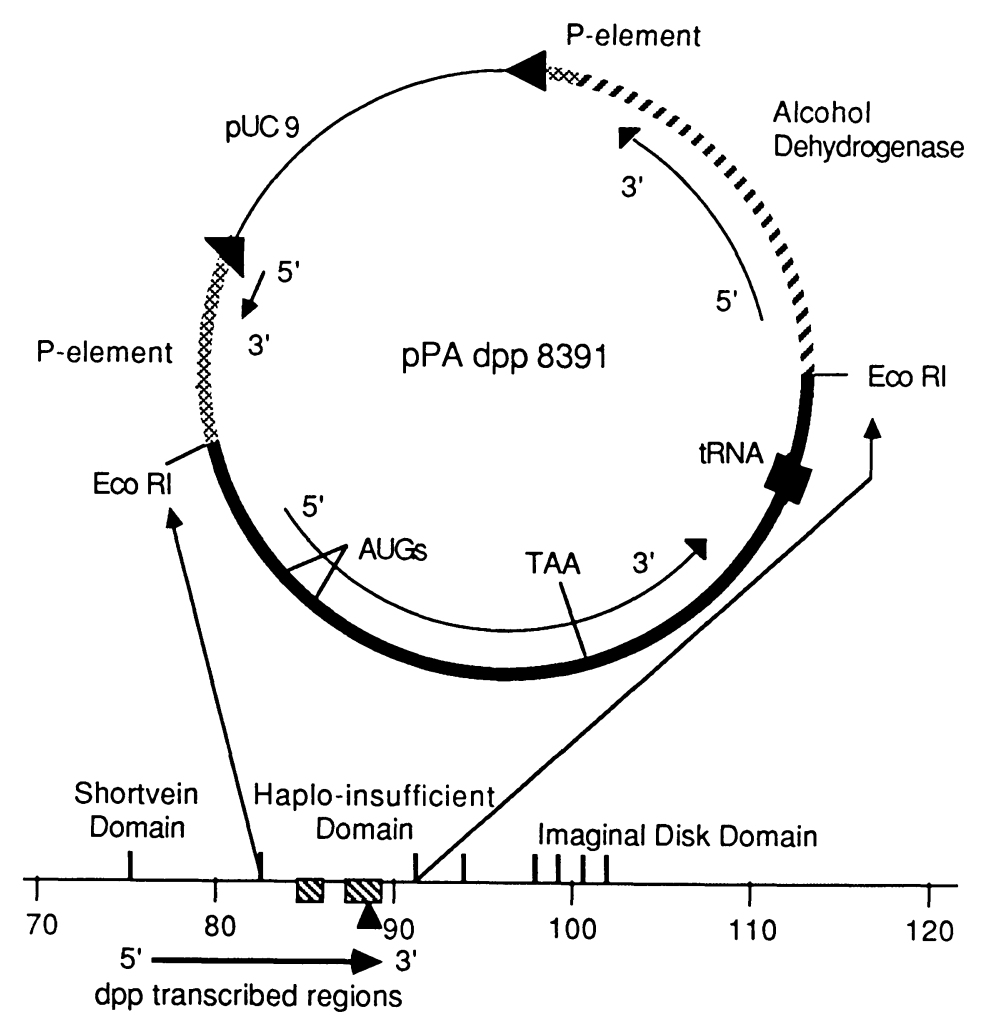

\author{
I = Eco RI site \\ $\mathbb{N}=$ Protein coding exons \\ $\Delta=$ TGF-beta homolgy
}

Figure 1. Construction of the $d p p$ transposon plasmid. An 8-kb EcoRI fragment was isolated from a genomic phage clone of $d p p$ DNA and inserted into a unique EcoRI site of P-element transposon plasmid pPA-l. The origin of the $d p p$ fragment is shown relative to the three domains of the $d p p$ gene (bottom). The numbers are kilobases of DNA along a chromosomal walk used to isolate the $d p p$ gene. The orientation of transcription from three Drosophila promoters, the adh promoter, the P-element promoter, and a putative dpp promoter, are shown for pPAdpp8391; the dpp insert in pPAdpp 9183 is oriented in the opposite direction. The location of the two inframe AUG codons and the termination codon reported by Padgett et al. (1987) are shown on the transcribed portion of the $d p p$ sequences. One tRNA ${ }^{\text {Tyr }}$ is also present on the $d p p$ fragment. The P-element terminal repeats that mediate integration into the Drosophila chromosome are shown by the large arrows on the circle. are wild type for dpp ("none", for no transposon; Table 2 ), the male progeny do not inherit the paternal $X$ chromosome with the duplication of $d p p$. Therefore, males

Table 1. dpp8391 transposon lines

\begin{tabular}{lccc}
\hline $\begin{array}{l}\text { Plasmid } \\
\text { injected }\end{array}$ & $\begin{array}{l}\text { Transposon } \\
\text { name }\end{array}$ & $\begin{array}{l}\text { Mapped to } \\
\text { chromosome }\end{array}$ & $\begin{array}{l}\text { Location on } \\
\text { polytene } \\
\text { chromosome }\end{array}$ \\
\hline pPAdpp9183 & TnMH1 & 3 & $64 \mathrm{E}, \mathrm{F}$ \\
pPAdpp8391 & TnJW1 & 3 & $93 \mathrm{~A}, \mathrm{~B}$ \\
pPAdpp8391 & TnJW2 & 2 & 23D \\
pPAdpp8391 & TnJW3 & 2 & $46 \mathrm{C}$ \\
pPAdpp8391 & TnJA1 & X & n.d. \\
pPAdpp8391 & TnJA2 & 3 & $82 \mathrm{~A}$ \\
pPAdpp8391 & TnJA3 & 3 & $79 \mathrm{E}$ \\
\hline
\end{tabular}

Progeny from seven different injected embryos survived the ethanol selection for the $d d h$ gene. Single survivors from each of the seven embryos were used to establish a line of the transposon. The flies were crossed to an $a d h$-null stock carrying the dominantly marked balancer chromosomes Cy0 and TM2. Ethanol-resistant progeny that were $C y$ and $U b x$ were backcrossed to an $a d h$-null stock, and the segregation of the ethanol resistance was followed relative to the dominantly marked second and third chromosomes. After assignment of the transposon to a specific chromosome, a balanced stock of each of the transposon lines was established. The specific chromosomal location of the transposons was determined by preparation of salivary gland polytene chromosomes and hybridization with a ${ }^{3} \mathrm{H}$-labeled $d p p$ probe (Pardue and Gall 1975). Each of the lines used carried a single insertion of the transposon, as determined by in situ hybridization and by genomic Southern blot analysis of the DNA (data not shown). that inherit the paternal $d p p^{H i n}$ allele die as embryos because the one intact copy of the $d p p$ gene they inherit is insufficient for embryonic development.

When the males with the X-linked duplication and the $d p p^{H i n}$ alleles were mated to females from the transgenic lines, the presence of the transposon-bearing chromosome permitted recovery of viable fertile males in spite of the fact that these males did not inherit the X-linked duplication of $d p p$ (Table 2). The quantitative nature of the rescue by the transposon was determined by scoring the number of progeny in each of the progeny classes that did not involve transposon rescue of the mutant allele, calculating the total progeny in these classes, and dividing by the number of such classes to arrive at the average size of an individual progeny class. These are the denominators in Table 2. The numerator is the actual number of adult male flies scored in the experimental class, that is, the male progeny with the $d p p^{\text {Hin }}$ allele.

Each of the seven transgenic lines was able to rescue the haplo-lethality of five different $d p p$ mutant alleles, including $\mathrm{Df}(2 \mathrm{~L}) \mathrm{DTD} 2$, a large multigenic deletion that removes the entire $d p p$ gene and portions of the chromosome on either side of $d p p . d p p^{\text {Hin37 }}, d p p^{\text {Hin46 }}, d p p^{\text {Hin47, }}$ and $d p p^{H i n 61}$ all contain a deletion or rearrangement breakpoint in their DNAs between coordinates 83 and 91 on the DNA (Gelbart et al. 1985). For the five rescued alleles, the actual number of rescued flies ranged from 66 to $100 \%$ of the expected number, based on sibling progeny classes. Although the different chromosomal locations of the transposons were expected to affect their level of expression and, thereby, their potency in rescuing mutant alleles, none of the transposon lines was significantly better or worse than the others in re- 
scuing the haplo-lethality of one copy of the $d p p$ gene.

For the transposons integrated on the $\mathrm{X}$ or third chromosomes, the experiments were designed so that the transposon segregated away from a dominant visible marker. Only one of the chromosomes in the transgenic mothers carried the transposon, and the homologous chromosome was dominantly marked with either Bar $(B)$ eyes on the $\mathrm{X}$, or $U b x$ or Tubby $(T b)$ on the third. In these cases, male progeny with the $d p p^{\text {Hin }}$ allele were viable only when they inherited the transposon-bearing maternal chromosome $\left(\mathrm{Ubx}^{+}, \mathrm{Tb}^{+}\right.$, or $\left.\mathrm{B}^{+}\right)$. We did not recover males with the $d p p$ mutation and the dominant marker $(U b x, T b$, or $B)$ beyond the expected numbers of escapers from the haplo-lethality (Table 2 ).

The one exception was the failure of any of the transposon lines to rescue $d p p^{H i n 47}$. However, this allele also failed to be rescued by $\mathrm{Dp}$ (2L)DTD48, a duplication of polytene bands $22 \mathrm{E} 2-4$ to $23 \mathrm{Al}$ containing the entire $d p p$ gene, one lethal complementation group proximal to $d p p$, and more than $70 \mathrm{~kb}$ of DNA, undefined genetically, distal to $d p p$. Because the duplication of the entire $d p p$ gene did not yield $d p p^{H i n 47}$ adult progeny, it is not surprising that the small piece of the $d p p$ gene on the transposon was unsuccessful. We expect that there may be some other lesion on the chromosome bearing $d p p^{\text {Hin } 47}$ that precludes viability with $\mathrm{Dp}(2 \mathrm{~L}) \mathrm{DTD} 48$ or the transposon $d p p 8391$.

\section{Rescue of the ventralized null phenotype}

We concluded that one intact $d p p$ gene and the transposon with only $8 \mathrm{~kb}$ of the $d p p$ gene were able to provide all the $d p p$ product required for correct development and survival to adulthood. However, mutations in $d p p$ block at least three aspects of development: (1) embryonic dorsal-ventral pattern formation (Irish 1986), (2)

Table 2. Transposon rescue of $\mathrm{dpp}^{\text {Hin }}$ alleles

\begin{tabular}{|c|c|c|c|c|c|c|c|c|}
\hline \multirow[b]{4}{*}{$d p p^{\text {Hin }}$ allele } & \multicolumn{8}{|c|}{ Actual number of adult male progeny with the $d p p^{\mathrm{Hin}}$ allele } \\
\hline & \multicolumn{8}{|c|}{ expected size of this progeny class based on the number of siblings } \\
\hline & \multicolumn{8}{|c|}{$d p p$ transposon line tested } \\
\hline & none $^{\mathbf{a}}$ & TnMH1 ${ }^{\mathrm{b}}$ & TnJWlb & TnJW2c & TnJW3 ${ }^{c}$ & TnJAld & $\operatorname{Tn} J A 2^{b}$ & $\operatorname{Tn} J A 3^{b}$ \\
\hline \multirow{2}{*}{ Df(2L)DTD2 } & \multirow{2}{*}{$2 / 140$} & $56 \mathrm{Ubx}^{+} / 80$ & $50 \mathrm{Ubx}^{+} / 77$ & \multirow{2}{*}{$92 / 84$} & \multirow{2}{*}{$79 / 100$} & $68 B^{+} / 68$ & $60 \mathrm{~Tb}^{+} / 57$ & $58 T b^{+} / 61$ \\
\hline & & $0 U b x / 80$ & $5 U b x / 77$ & & & $4 B / 68$ & $0 \mathrm{~Tb} / 57$ & $0 T b / 61$ \\
\hline \multirow{2}{*}{$d p p^{\text {Hin37 }}$} & \multirow{2}{*}{$0 / 82$} & $134 U b x^{+} / 110$ & $56 \mathrm{~Tb}^{+} / 64$ & \multirow{2}{*}{$97 / 116$} & \multirow{2}{*}{$63 / 74$} & \multirow{2}{*}{ n.d. } & $75 U b x^{+} / 67$ & $87 \mathrm{Ubx}^{+} / 115$ \\
\hline & & $0 U b x / 110$ & $0 \mathrm{~Tb} / 64$ & & & & $0 U b x / 67$ & $0 U b x / 115$ \\
\hline \multirow{2}{*}{$d p p^{\operatorname{Hin} 46}$} & \multirow{2}{*}{$0 / 175$} & \multirow{2}{*}{ n.d. } & $124 \mathrm{~Tb}^{+} / 160$ & \multirow[t]{2}{*}{$339 / 359$} & \multirow[t]{2}{*}{$339 / 309$} & \multirow[t]{2}{*}{ n.d. } & $188 \mathrm{~Tb}^{+} / 132$ & \multirow[t]{2}{*}{ n.d. } \\
\hline & & & $0 \mathrm{~Tb} / 160$ & & & & $0 \mathrm{~Tb} / 132$ & \\
\hline$d p p^{\text {Hin } 47}$ & $0 / 114$ & $0 / 63$ & $1 / 191$ & $0 / 97$ & $0 / 143$ & $0 / 73$ & n.d. & n.d. \\
\hline \multirow[t]{2}{*}{$d p p^{\operatorname{Hin} 48}$} & \multirow[t]{2}{*}{$0 / 103$} & \multirow[t]{2}{*}{ n.d. } & \multirow[t]{2}{*}{ n.d. } & \multirow[t]{2}{*}{$138 / 135$} & \multirow[t]{2}{*}{$98 / 119$} & $57 B^{+} / 59$ & \multirow[t]{2}{*}{ n.d. } & \multirow[t]{2}{*}{ n.d. } \\
\hline & & & & & & $0 B / 59$ & & \\
\hline \multirow[t]{2}{*}{$d p p^{\text {Hin61 }}$} & \multirow[t]{2}{*}{$0 / 102$} & $80 U b x^{+} / 87$ & \multirow[t]{2}{*}{ n.d. } & \multirow[t]{2}{*}{$105 / 158$} & \multirow{2}{*}{$78 / 76$} & $68 B^{+} / 55$ & $117 \mathrm{Ubx}^{+} / 90$ & $68 \mathrm{~Tb}^{+} / 82$ \\
\hline & & $0 U b x / 87$ & & & & $2 B / 55$ & $0 U b x / 90$ & $0 \mathrm{~Tb} / 82$ \\
\hline
\end{tabular}

Fathers carried a wild-type duplication of the $d p p$ gene on the $\mathrm{X}$ chromosome and any of six different $d p p^{\text {Hin }}$ alleles as listed in the first column. Mothers were from the $a d h$-null stock (two wild-type copies of the $d p p$ gene but no additional duplications of the $d p p$ gene, labeled "none") or from each of the seven lines carrying the $d p p$ transposon in addition to two wild-type copies of the $d p p$ gene. The phenotypes of the progeny were scored with respect to the sex and visible phenotypes imparted by dominant markers used in the cross. The $\mathrm{Cy}^{+}$males inherited the $d p p^{H i n}$ allele in the absence of the $\mathrm{X}$-linked $d p p$ duplication. Their viability was used as a criterion for $d p p$ function from the transposon. Df(2L)DTD2 removes polytene bands 22D4-5 to 23B1-2, which include the $d p p$ gene. This deficiency is lethal when heterozygous to a wild-type chromosome for this region, although there is a small amount of leakiness associated with the haplo-lethality ( 2 survivors when 140 were expected). With the exception of $d p p^{\text {Hin } 48}$, in which no DNA lesion has yet been mapped, the four $d p p^{\text {Hin }}$ alleles are all associated with defects in the DNA in the embryonic region (Gelbart et al. 1985).

$$
\begin{aligned}
& \frac{\text { a } \mathrm{Dp}(2 ; 1) \mathrm{G} 146}{\mathrm{Y}} ; \frac{d p p^{\text {Hin }}}{\mathrm{CyO}} \times a d h^{f n 6}, p r, c n \\
& \frac{\mathrm{b} \mathrm{Dp}(2 ; 1) \mathrm{G} 146}{\mathrm{Y}} ; \frac{d p p^{H i n}}{\mathrm{CyO}} \times a d h^{f n 6}, p r, c n ; \frac{\mathrm{Tn} d p p 8391}{\mathrm{TM} 2(U b \mathrm{U}) \text { or TM } 6, B(T b)} \\
& \frac{\mathrm{c} \mathrm{Dp}(2 ; 1) \mathrm{G} 146}{\mathrm{Y}} ; \frac{d p p^{H i n}}{\mathrm{CyO}} \times a d h^{f n 6}, p r, c n, \mathrm{Tn} d p p 8391 \\
& \frac{\mathrm{d} \mathrm{Dp}(2 ; 1) \mathrm{G} 146}{\mathrm{Y}} ; \frac{d p p^{H i n}}{\mathrm{CyO}} \times \frac{\mathrm{Tn} d p p 8391}{\mathrm{FM} 7 \mathrm{a}(B)} ; a d h^{f n 6}, p r, c n
\end{aligned}
$$


larval growth and survival (Segal and Gelbart 1985), and (3) growth of imaginal disk tissues (Spencer et al. 1982). We wished to determine which of the multiple functions of $d p p$ were carried out by products expressed by the transposon.

To address this question, we produced animals heterozygous for two deletions of the $d p p$ gene. These deletions should eliminate $d p p$ products from both endogenous copies of the gene. The only intact copies of $d p p$ protein-coding sequences in such animals would be supplied by the transposon. The deletions of $d p p$ used in these experiments were Df(2L)DTD2, a large multigenic deletion that removes the entire $d p p$ gene, and $\mathrm{Df}(2 \mathrm{~L}) d p p^{H i n 61}$, an internal deletion of $2.5 \mathrm{~kb}$ in the Hin domain (Spencer et al. 1982; Gelbart et al. 1985). The $\mathrm{Df}(2 \mathrm{~L}) d p p^{H i n 61}$ allele does not remove DNA from the shortvein and imaginal domains, but it does behave genetically as a null allele for these functions in failing to complement mutant shortvein and disk $d p p$ alleles. The deletion removes approximately 300 amino acids from the carboxyl terminus of the only reported proteincoding sequences at $d p p$ (Padgett et al. 1987). The ideal null allele would remove all $d p p$ DNA without affecting flanking genes, but in the absence of such a deletion, we have used $d p p^{H i n 61}$ as the largest internal deletion of $d p p$ sequences that behaves genetically as a null allele.

When the endogenous copies of $d p p$ were eliminated by the two deletion alleles, the dead embryos had a completely ventralized cuticle (Gelbart et al. 1985). The ventral denticle belts wrapped entirely around the circumference of the larval cuticle formed during embryogenesis (Fig. 2b). Development of the extreme anterior and posterior ends of the embryo was affected severely: The posterior spiracles and the head structures, most prominently the cephalopharyngeal skeleton, were absent. Frequently, the most posterior denticle belts defined an unusual posterior invagination of the cuticle. There was usually a reduction in the number of segments as defined by the number of denticle belts.

The presence of one copy of the $d p p$ transposon was sufficient to provide a normal ventral pattern of denticle belts (Fig. 2c). However, the extreme anterior and posterior ends were still defective: The anterior end of the cuticle was often open as if head involution had not occurred, and, frequently, the posterior spiracles were not everted. The pattern of dorsal hairs was more variable than in a wild-type embryo; the anterior rows of thick dorsal hairs were usually present, but the broad areas normally covered with slender hairs were frequently bare. The cuticular phenotype was similar to that observed in animals with one Hin allele and one wild-type allele of $d p p$ (Fig. 2d).

\section{Two copies permit larval hatching}

We quantitated the effect of the transposon on successful embryonic development and hatching of the larval form. Eggs from matings that would produce $25 \%$ $d p p$-null embryos with zero, one, or two copies of the transposon were collected, counted, and permitted to

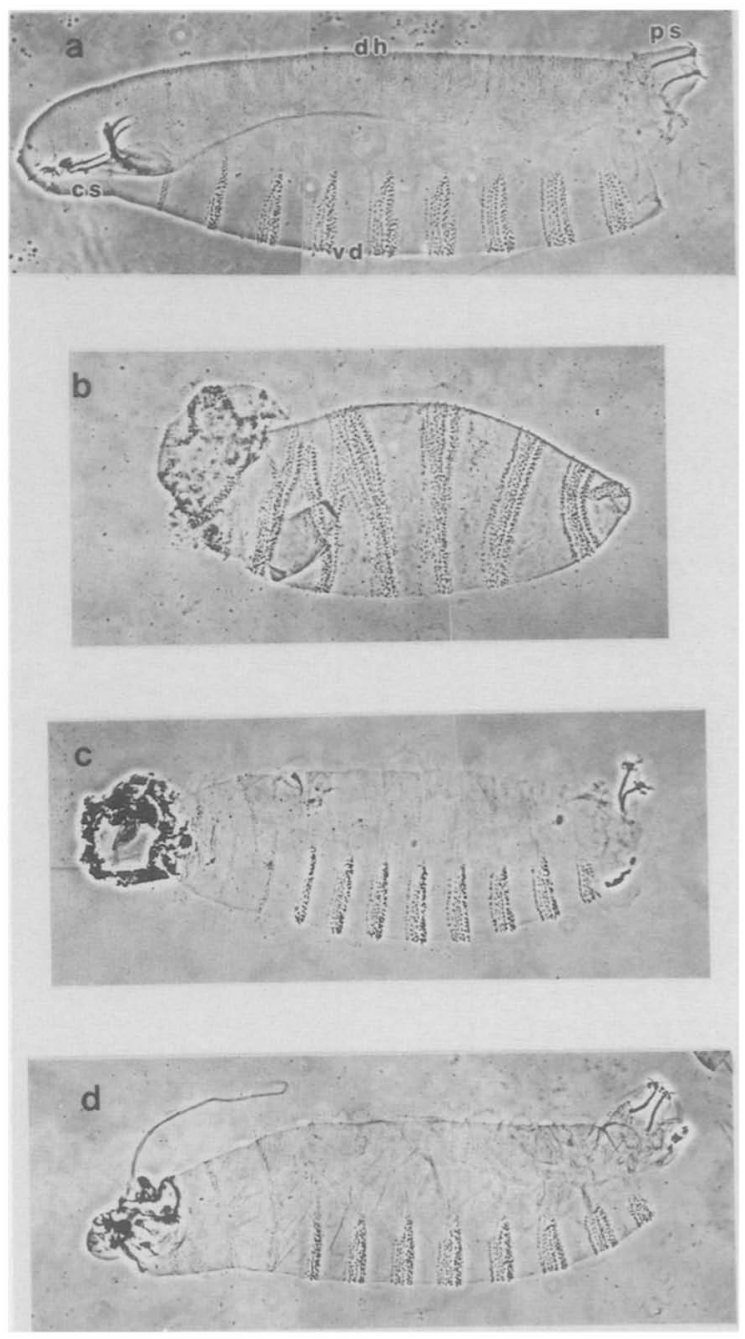

Figure 2. Cuticular phenotypes of wild-type, $d p p$ mutant, and rescued animals. Cuticles were prepared as described in Experimental procedures from unhatched embryos of wild-type $(a)$, $d p p$-null $(b), d p p$-null plus one copy of transposon $d p p 8391(c)$, and $d p p^{H i n} /+(d)$ animals. The dorsal surface is at the top of each panel, and anterior to the left of each panel. The wild-type structures of the ventral denticle belts (vd), the dorsal hairs (dh), the cephalopharyngeal skeleton (cs), and the posterior spiracles (ps) are noted in $a$. All of these structures are affected in the dpp-null cuticle, with the dorsal hairs, cephalopharyngeal skeleton, and posterior spiracles absent from the mutant cuticle and the ventral denticle belts extending completely around the circumference of the animal. Much of the wild-type pattern is restored in $c$ by one copy of transposon dpp8391, including the ventral localization of the denticle belts and the appearance of posterior spiracle structures. Elements of the cephalopharyngeal skeleton are externalized, and the anterior end of the cuticle appears to be open. These animals fail to hatch from the eggshell.

hatch. The chorion from the unhatched eggs and embryos was removed $48-72 \mathrm{hr}$ after egg laying, and the extent of development was scored. Many of the unhatched eggs appeared to be undeveloped and exhibited the uniform opaqueness of an unfertilized egg in both experimental and control crosses (Table 3; undeveloped 
eggs). This was probably due to the highly inbred nature of the lines used in the experiment.

The remainder of the unhatched eggs contained dead embryos, with clear evidence of segmentation and cuticle formation even in the crosses that generated $d p p$ null animals with ventralized cuticles. The number of the unhatched, but developed, dead embryos was used as an indicator of the lethality due to the mutations in the $d p p$ gene. By this criterion, null embryos with one copy of the transposon failed to hatch, independent of whether the transposon was derived from the maternal or paternal genome (Table 3). The embryos hatched to larvae when two copies of the transposon were present (Table 3). Thus, in the absence of the endogenous genes,

Table 3. Transposon rescue of the dpp-null phenotype

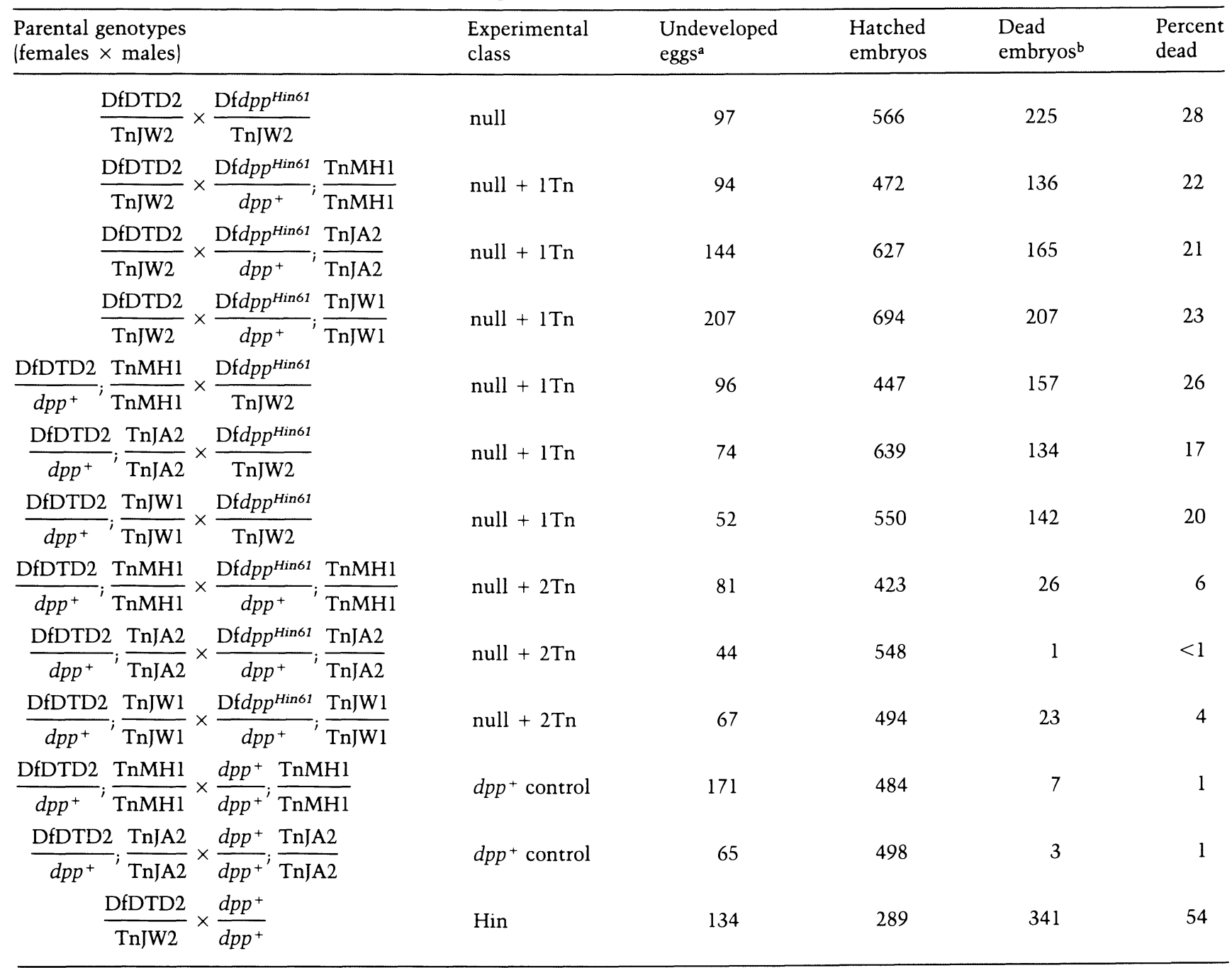

Transposon rescue of the $d p p$-null phenotype. The $d p p$ alleles used represent null mutations normally haplo-lethal but viable in the presence of one copy of the $d p p$ transposon, as indicated here in the parental genotypes. The experimental class represents the $25 \%$ of the progeny that inherit both null alleles and from zero to two copies of the transposon. All of the other progeny classes generated in these crosses are adult viable. Control crosses $\left(d p p^{+}\right)$with the $d p p^{H i n 61}$ allele gave results comparable to those shown for DfDTD2. The large number of undeveloped eggs found in both experimental and control crosses are believed to be due to the inbred stocks used and independent of the specific transposon line or $d p p$ alleles present. A binomial likelihood ratio test was used to examine the proportions of hatched versus dead embryos. Five out of six crosses that generated "null $+1 \operatorname{Tn}^{\prime}$ progeny produced significant decreases in the number of dead embryos $(p<0.005)$. The maternally derived TnMH1 produced no significant effect on the embryonic lethality in one copy $(p=0.15)$. Among the five null $+1 \mathrm{Tn}$ crosses that provided improved embryonic viability, there was no significant difference in the amount of viability provided by different transposon lines or by maternal vs. paternal inheritance of the transposon $(p=0.1-0.5)$.

a Undeveloped eggs showed no development of cuticle or internal structures upon mechanical removal of the chorion $48-72 \mathrm{hr}$ after egg lay; the appearance was the same as unfertilized eggs.

b Dead embryos were removed mechanically from the chorion $48-72 \mathrm{hr}$ after egg lay; ventral denticle belts were scored as an indication of cuticle formation. 
the $d p p$ sequences on the transposons provided sufficient product to permit embryonic development to the larvae. As in the case of the intact $d p p$ gene, two copies of the transposon were necessary to provide enough of the embryonically required $d p p$ products.

In experiments in which all progeny classes could be unambiguously identified at the adult stage by dominant visible markers, the hatched animals in the progeny classes with two copies of the transposon did not develop to the pupal or adult forms. Although the $d p p$ sequences on the transposon rescued the embryonic lethal phenotype, the rescued larvae did not develop further. We observed the same arrested larval development that was observed in animals trans-heterozygous for mutant $d p p^{s h v}$ alleles (Segal and Gelbart 1985).

We expected that a specific transposon might not provide sufficient product in two copies because the level of expression from a transposon may be reduced, compared with its parent gene, depending on the chromosomal position of the transposon (Hazelrigg et al. 1984). However, for the three transposon lines tested, all permitted hatching to larvae in two copies. The requirement for, and sufficiency of, two copies suggested that the quantitative level of expression of the $d p p$ product from the transposon was similar to that from the intact gene.

\section{Rescue of recessive embryonic lethals}

The other $d p p$ alleles that affect embryonic development are the recessive embryonic lethals (Spencer et al. 1982). These alleles must involve subtle changes in the DNA, as none of them is associated with a gross chromosomal rearrangement at $d p p$. These mutant alleles behave differently from the $d p p^{H i n}$ alleles in that the recessive alleles are fully viable in the presence of one normal copy of $d p p$. However, the embryonic lethal alleles are similar to the $d p p^{H i n}$ alleles in two respects: The embryonic lethal phenotype of an animal trans-heterozygous for two recessive lethal alleles is similar to the phenotype of an animal with one $d p p^{H i n}$ allele and one wild-type $d p p$ allele (Irish 1986); and, like the $d p p^{H i n}$ alleles, most of the embryonic lethal alleles are also defective for shortvein and disk functions, as evidenced by a lack of genetic complementation. The recessive embryonic lethal alleles may be reduced, but not totally null, for the same $d p p$ function eliminated by haplo-lethal alleles. If the recessive lethal alleles affect the same $d p p$ product or products as do the haplo-lethal alleles, the $d p p$ sequences on the transposon should rescue the embryonic lethality.

Two of the embryonic recessive lethal alleles, $d p p^{e 87}$ and $d p p^{I C 38}$, were fully rescued to adulthood by one copy of the transposon in flies in which the other endogenous copy of $d p p$ was deleted by Df(2L)DTD2 (Table 4). Unlike the other embryonic recessive lethal alleles, these two alleles complement lesions in both the shortvein and disk regions of $d p p$. The only $d p p$ alleles they fail to complement are the other recessive embryonic lethal alleles. Because they were fully rescued by the transposon, we conclude that these mutations disrupt only the $d p p$ product or products expressed from the DNA on the transposon.

The embryonic lethality in animals hemizygous for two other recessive lethal alleles, $d p p^{\text {hinrt }}$ and $d p p^{\text {hinr } 56}$, was overcome by the presence of two copies of the transposon (Table 4). Progeny heterozygous for the two recessive lethal $d p p$ alleles were also fully rescued from the embryonic lethality by the presence of two copies of the transposon. However, the rescued animals did not survive to adults. In two cases, the number of dead pupae was approximately equal to the number of animals in the sibling progeny classes. These two cases involved the $d p p^{\text {hinrt }}$ allele, either as a hemizygote or as a heterozygote with $d p p^{\text {hinr } 56}$, in the presence of two copies of the transposon TnIA2 (Table 4). The dead pupae exhibited a specific phenotype. The anterior portion of the pupal case was usually empty, and the posterior half was filled with amorphous tissue but not any adult cuticle. A similar phenotype was observed for severe (class V) imaginal disk $d p p$ alleles.

Crosses that generated progeny hemizygous for $d p p^{h i n r 56}$ with two copies of the transposon, or $d p p^{h i n r} 4$ with two copies of transposon TnMH1, showed no significant pupal lethality (Table 4). From experiments in which all adult progeny classes could be identified unambiguously, we knew that these genotypes did not survive to adulthood. Therefore, these progeny classes must die as larvae. Although we have not examined the larval lethality quantitatively, we observed animals with arrested larval growth similar to that reported for the shortvein mutant alleles (Segal and Gelbart 1985).

\section{The transposon does not affect adult viable imaginal and shortvein phenotypes}

The larval and early pupal lethality observed in the presence of two copies of the transposon indicated that the transposon did not provide either shortvein or disk $d p p$ functions. To test this hypothesis more fully, we generated flies that were hemizygous for a number of shortvein or imaginal disk mutant alleles by removing the other copy of $d p p$ with Df(2L)DTD2 and providing $d p p$ embryonic function with one copy of the transposon. In all cases, the shortvein and imaginal disk mutant phenotypes were observed, even for the phenotypically mildest shortvein $\left(d p p^{s l}\right)$ and disk $\left(d p p^{d-h o}\right)$ mutant alleles. A second copy of the transposon did not alleviate these mutant phenotypes.

\section{Discussion}

The technique of making transgenic Drosophila by P-element-mediated DNA transformation provides a way to assay the biological activity of specific DNA sequences in the whole animal. We have used this technique to show that a DNA fragment representing, at most, onefifth of the $d p p$ gene contains sufficient regulatory and structural sequences to rescue the ventralized embryonic phenotype and the embryonic lethality caused by a number of mutations in $d p p$ (Fig. 3). The $d p p$ function 
Table 4. Transposon rescue of the dpp recessive embryonic lethals

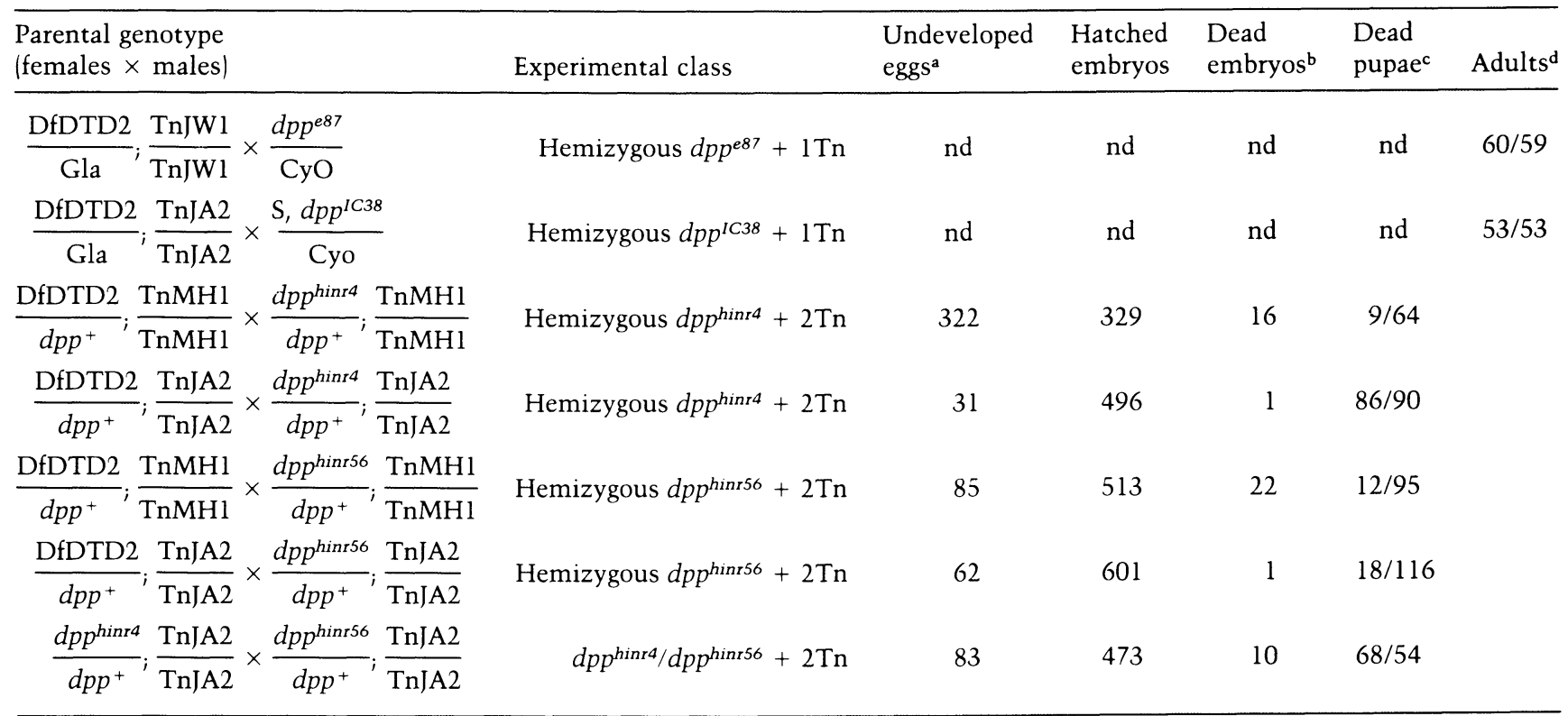

Transposon rescue of the $d p p$ recessive embryonic lethals. The $d p p$ alleles tested are recessive lethals as trans-heterozygotes with each other. The $d p p^{e 87}$ and $d p p^{I C 38}$ alleles complement $d p p$ imaginal disk and shortvein alleles, but $d p p^{\text {hinr } 4}$ and $d p p^{\text {hinr } 56}$ fail to complement most $d p p$ imaginal and shortvein alleles. The alleles were tested as hemizygotes by use of Df(2L)DTD2 to remove the entire $d p p$ gene. The proportions of hatched and dead embryos should be compared $d p p^{+}$control and $d p p$-null crosses in Table 3 . Adult phenotypes were scored only for the first two crosses, because the other five crosses did not include sufficient visible marker mutations on the chromosomes to permit unambiguous assignment to progeny classes.

${ }^{a}$ Undeveloped eggs showed no development of cuticle or internal structures upon mechanical removal of the chorion $48-72 \mathrm{hr}$ after egg lay; the appearance was the same as unfertilized eggs.

${ }^{b}$ Dead embryos were mechanically removed from the chorion $48-72 \mathrm{hr}$ after egg lay; ventral denticle belts were scored as an indication of cuticle formation.

${ }^{c}$ Number of dead pupae/expected number based on the number of sibling adult survivors.

${ }^{d}$ Number of adults in experimental class/expected number based on total adult progeny.

provided by the transposon rescues both the haplolethals and the recessive embryonic lethal alleles. The dominant lethality of the $d p p^{H i n}$ alleles had confounded the genetic assignment of the haplo-lethal and recessive embryonic lethals to the same function, but the observation that both are rescued by the same defined piece of DNA is consistent with the idea that both affect the same $d p p$ function. The difference in the genetic behavior of the two types of alleles is probably due to quantitative differences in the activity of the gene product produced by the mutant genes.

The line established with $\operatorname{Tn} M H 1$ carries the $d p p$ 83-91 EcoRI fragment in the opposite orientation relative to the adh gene on the transposon (Table 1). The orientation in the transgenic flies was confirmed by genomic Southern blotting. The orientation-independent rescue of the $d p p^{H i n}$ alleles is consistent with transcription of the $d p p$ sequences being driven from a promoter on the $d p p$ fragment and not from the P element or $a d h$ promoters. We have not eliminated the possibility that cryptic promoter sequences present on the transposon are driving the expression of $d p p$ transcription in either orientation. In addition, we have not determined whether the tRNA ${ }^{\mathrm{Tyr}}$ gene on the transposon has any role in the rescue. Because most of the Hin alleles used in this work map within the sequences transcribed into
mRNA products, and not near the tRNA sequences, we believe that the tRNA gene on the transposon is not responsible for the rescue of the $d p p$ mutant phenotypes. Experiments in progress to define the $d p p$ promoter and the RNA products made by the transposon will address these questions directly. It has been reported that four of the five $d p p$ transcripts initiate in the shortvein domain (Padgett et al. 1987). We predict that the fifth transcriptional initiation site is within the 83-91 fragment.

The haplo-insufficiency of one intact copy of the $d p p$ gene was rescued by one copy of the transposon, and the rescued animals survived to fertile, viable adults. We interpret this result to mean that there is a threshold quantity of $d p p$ product required in the early embryo and that one copy of the $d p p$ gene is incapable of supplying enough product. The $d p p$ sequences on the transposon encode this dosage-sensitive product and supply additional quantities sufficient to attain the threshold level. Once the embryonic functions are accomplished, the survival to adulthood requires that the larval and imaginal disk functions of $d p p$ are carried out. Because mutations in the imaginal disk and shortvein regions are strictly recessive in character, showing no haplo-insufficiency, it is likely that one intact copy of the $d p p$ gene is sufficient to provide product for the imaginal disk and shortvein functions of $d p p$. 
Mutant animals hemizygous for $d p p$ alleles that affect only the embryonic function of $d p p, d p p^{e 87}$, and $d p p^{I C 38}$ were also rescued to viable, fertile adults by the transposon (Fig. 3). We should note that the chromosome bearing $d p p^{I C 38}$ used in these studies carries the dominant mutation Star $(S)$ and the recessive mutation cinnebar $(c n)$; when this chromosome is used in complementation tests, the $d p p^{I C 38}$ allele complements imaginal and shortvein alleles. It has been reported that $d p p^{I C 38}$ on a recombinant chromosome that does not carry $S$ exhibits a temperature-sensitive lack of complementation with imaginal alleles (Irish 1986). We suspect that the transposon rescue to adults may be dependent on which $d p p^{I C 38}$ chromosome is used.

The effectiveness of the transposon $d p p$ sequences was observed in the $d p p$-null embryo. First, one copy of the transposon in a $d p p$-null animal restored much of the dorsal pattern of the cuticle formed during embryogenesis. The circumferential pattern of the denticle bands was replaced by an essentially normal ventral pattern of the bands. However, the cuticle was not restored to a wild-type appearance. In particular, the dorsal hairs did not occupy as broad an area of the cuticle, and the extreme anterior and posterior cuticular structures were not normal. It will be of interest to characterize the role of $d p p$ at the anterior and posterior poles of the embryo and to determine whether this function is coupled to the elaboration of dorsal-ventral pattern formation or whether it is a separate function of the $d p p$ product.

The cuticular phenotypes and the failure of the animals to hatch from the egg case indicated that the $d p p$ DNA on the transposon was as potent as, but no better than, one intact copy of the $d p p$ gene. In fact, one copy of the transposon did provide a small, but statistically significant, decrease in the number of dead embryos (Table 3). This is consistent with earlier observations that the haplo-lethality of $d p p^{H i n}$ alleles is leaky, yielding from 0 to $5 \%$ adult survivors, depending on the allele and its genetic background (Spencer et al. 1982). Thus, the transposon may give rise to approximately the same frequency of escapers as one intact copy of the $d p p$ gene, although, for reasons discussed below, the transposoninduced escapers from the embryonic lethality do not survive to adults. The best indication of the sufficiency of the transposon sequences was the successful embryonic development and larval hatching imparted to the otherwise $d p p$-null embryo by two copies of the transposon.

The $d p p^{H i n}$ alleles and the $d p p^{h i n r}$ recessive lethal alleles fail to complement lesions in the shortvein and imaginal disk functions of $d p p$. Therefore, although the earliest failure of the $d p p^{H i n}$ and $d p p^{h i n r}$ alleles is during embryogenesis, the lesions also disrupt the later functions of $d p p$. The death of the mutant transgenic organisms at the larval stage indicated that the transposon did not provide the $d p p$ functions needed for larval viability. Mutant alleles in the shortvein domain of $d p p$ cause larval lethality (Segal and Gelbart 1985). The conclusion that the portion of $d p p$ on the transposon is incapable of providing the $d p p$ functions disrupted by short-

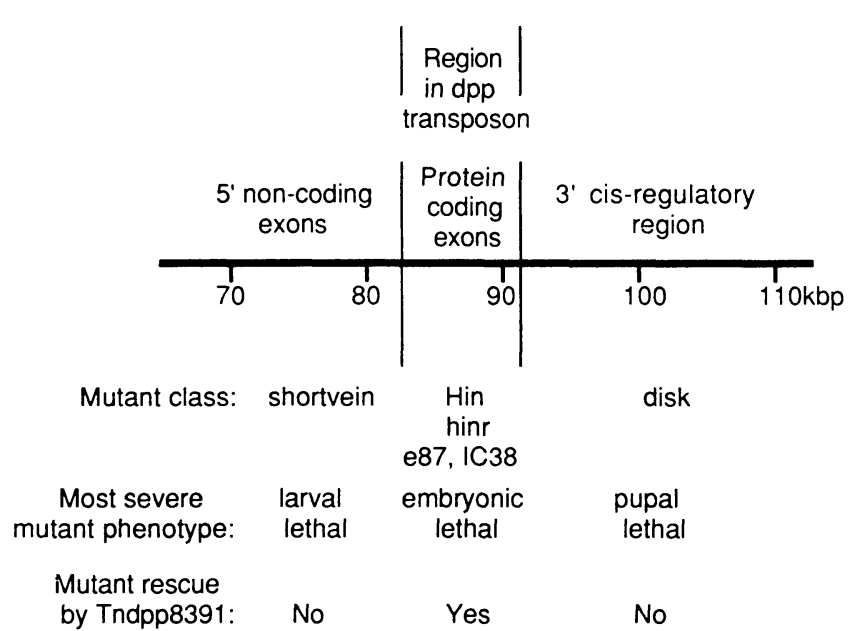

Figure 3. A model of the $d p p$ gene. The $d p p$ transposon rescue of $d p p$ mutant alleles supports a model consistent with previous molecular and genetic analysis of $d p p$. The portion of $d p p$ on the transposon $d p p 8391$ contains the protein-coding exons. Flanking this Hin region are sequences that include, on the left, 5 ' noncoding exons and unidentified promoter elements, and, on the right, $3^{\prime}$ cis-regulatory sequences. Lesions in each of these three domains produce lethal mutant phenotypes at three different times during development. Transposon dpp8391 rescues the embryonic lethality of lesions in the region that includes the protein-coding exons. The rescue results suggest that in addition to the $5^{\prime}$ noncoding exons in the shortvein region, there is at least one $d p p$ promoter and transcriptional initiation site in the Hin region. Also, the rescue results indicate that the $d p p$ regulatory sequences on either side of the proteincoding region are not required for the embryonic function that produces correct dorsal-ventral pattern and hatching to the larval form. Transposon $d p p 8391$ does not rescue mutations in the shortvein and disk regions of $d p p$. The $5^{\prime}$ and $3^{\prime} d p p$ regulatory elements must be necessary for proper $d p p$ expression during organogenesis, which is essential for viability of the larvae, and for proper $d p p$ expression in the imaginal disks.

vein mutant alleles is consistent with the fact that the transposon does not contain any of the $d p p$ sequences in which shortvein mutant lesions have been mapped.

The survival to pupae was unexpected because the transposon does not contain sequences from the shortvein region. One explanation is that the $d p p^{\text {hinrt }}$ recessive embryonic lethal allele is hypomorphic but not completely null for $d p p$ shortvein function. When the hypomorphic allele is present, sufficient shortvein function is provided by the mutant gene to permit larval viability and survival to pupae. The animals die as early pupae with no evidence of adult cuticle formation, a phenotype very similar to that caused by the most severe lesions in the imaginal disk domain of $d p p$ (Spencer et al. 1982). Neither the recessive embryonic lethal allele nor the $d p p$ sequences on the transposon are able to provide the $d p p$ imaginal functions necessary for growth of the imaginal disks.

However, the observation that only one of the two transposon lines allowed survival to pupae with the hemizygous $d p p^{\text {hinr }}$ allele raises the intriguing possibility that the product encoded on the transposon may be very 
similar to that required for larval functions associated with the shortvein domain. We have raised the point that different transposon lines can be expected to produce quantitatively different levels of product because of position effects at the site of insertion. For those differences to be seen, the biological response has to be sensitive to the level of product. For the most part, we have not seen differences in the biological responses to the transposon, but the sensitivity may not have been high enough. The rescue to pupation by transposon line $\operatorname{Tn} J A 2$ is an indication that the level of expression from this transposon may be sufficiently high to provide product for the larval viability functions of $d p p$. Although the transposon does not contain any of the expected regulatory sequences from the shortvein domain or the untranslated $5^{\prime}$ exon from the shortvein domain (Padgett et al. 1987), all of the known protein-coding sequences from the $d p p$ gene are within the sequences on the transposon (Fig. 3). Thus, with enough copies being expressed at sufficiently high levels, some of the larval viability functions may be restored by the transposon.

In the early embryo, $d p p$ may act in concert with a number of other Drosophila genes to establish or maintain the dorsal-ventral field of positional information, or $d p p$ may respond to this field to specify the developmental fate of the cells along the dorsal-ventral axis. The identification of the portion of the $d p p$ gene that exhibits the early embryonic function and the opportunity to assay the biological activity of this portion of the gene permit further molecular genetic manipulation of the $d p p$ DNA sequences. The goal of these studies will be to define the molecular interactions between $d p p$ and other components of dorsal-ventral pattern formation in the Drosophila embryo.

The $d p p 8391$ transposon is not competent to provide the $d p p$ product or products required for larval viability and growth of imaginal disk cells (Fig. 3). The dpp DNA fragment on the transposon is from the center of the $d p p$ gene. There are at least $10 \mathrm{~kb}$ of DNA in the shortvein domain to one side and more than $20 \mathrm{~kb}$ of DNA in the imaginal disk domain to the other side of the 83-91 fragment. The complementation patterns of mutant alleles could be explained if these flanking sequences contained cis-regulatory elements that acted upon the gene product encoded on the 83-91 fragment. For example, the reported cDNA structure indicates that alternate promoters and transcript initiation sites may reside in the shortvein domain, even though the predicted protein-coding sequence is contained entirely within the 83-91 fragment (Padgett et al. 1987). The functional dissection of the dpp gene will be extended to the shortvein and imaginal disk domains to identify sequences from these regions that are essential for $d p p$ function in larval viability and imaginal disk growth.

\section{Experimental procedures}

\section{Genetic stocks}

Isolation of the $d p p$ mutant alleles has been described previously (Gelbart 1982; Spencer et al. 1982; Segal and Gelbart
1985; Irish 1986). The TM6,B chromosome was described by Craymer (1984). All other genetic markers are described in Lindsley and Grell (1968).

\section{DNA manipulation}

Isolation and purification of plasmid DNA, restriction enzyme reactions, and DNA ligations were performed as described in Maniatis et al. (1982). Bacterial transformations into DHl were performed as described by Hanahan (1983).

\section{DNA transformation}

Preblastoderm Drosophila embryos null for the adh gene were injected with DNAs from the plasmid pPAdpp839l and from plasmid p25.7wc (Karess and Rubin 1984). The latter provides P-element transposase to catalyze integration of pPAdpp8391 but is unable to integrate itself. The animals that survive to adults are individually mated to flies null for $a d h$, and the resulting progeny are screened for the transposon bearing the wild-type $a d h$ gene by tests for survival after a 24-hr exposure to $6 \%$ ethanol (Vigue and Sofer 1976). Individual survivors are used to establish transgenic lines by being mated to the $a d h$ null stock.

\section{Cuticle preparation}

Unhatched embryos were dechorionated for $5 \mathrm{~min}$ in $50 \%$ bleach or by hand. The vitelline membrane was removed by a 10-min exposure to $10 \mathrm{ml}$ of heptane : $9 \mathrm{ml}$ of methanol : $1 \mathrm{ml}$ of $0.5 \mathrm{M}$ EDTA at $\mathrm{pH} 8.0$. The embryos were treated with $4: 1$ glacial acetic acid : glycerol at $65^{\circ} \mathrm{C}$ for $12 \mathrm{hr}$ and mounted in Hoyer's mounting medium (Van der Meer 1977).

\section{Acknowledgments}

We appreciate the assistance of Judith Austin and Julie Weeks with injections, Marianne Dooley with embryo counts, and Mitch Marti with stock maintenance. We thank Dr. Norman Drinkwater for advice on the statistical analysis and use of his computer program, and Dr. William F. Dove for a critical reading of the manuscript. This work was supported by grants POl-GM35099-01 to J.F. Crow and P30-CA07175 to H.C. Pitot from the National Institutes of Health.

\section{References}

Anderson, K.V. and C. Nusslein-Volhard. 1984. Genetic analysis of the dorsal/ventral embryonic pattern in Drosophila. In Pattern formation (ed. G.M. Malacinski and S.V. Bryant), pp. 269-289. Macmillan, New York.

Anderson, K.V., L. Bokla, and C. Nusslein-Volhard. 1985a. Establishment of dorsal-ventral polarity in the Drosophila embryo: The induction of polarity by the Toll gene product. Cell 42: 791-798.

Anderson, K.V., G. Jurgens, and C. Nüsslein-Volhard. 1985b. Establishment of dorsal-ventral polarity in the Drosophila embryo: Genetic studies on the role of the Toll gene product. Cell 42: 779-789.

Craymer, L. 1984. New mutants. Dros. Inf. Serv. 60: 234.

Gelbart, W.M. 1982. Synapsis-dependent allelic complementation at the decapentaplegic gene complex in Drosophila melanogaster. Proc. Natl. Acad. Sci. 79: 2636-2641.

Gelbart, W.M., V.F. Irish, D. St. Johnston, F.M. Hoffmann, R. Blackman, D. Segal, L.M. Posakony, and R. Grimalia. 1985. The decapentaplegic gene complex in Drosophila melano- 
gaster. Cold Spring Harbor Symp. Quant. Biol. 50: 119125.

Hanahan, D. 1983. Studies on transformation of Escherichia coli with plasmids. J. Mol. Biol. 166: 557-580.

Hazelrigg, T., R. Levis, and G.M. Rubin. 1984. Transformation of white locus DNA in Drosophila: Dosage compensation, zeste interaction, and position effects. Cell 36: 469-481.

Irish, V.F. 1986. "Embryonic function of the decapentaplegic gene complex in Drosophila melanogaster." Ph.D. thesis, Harvard University.

Karess, R.E. and G.M. Rubin. 1984. Analysis of P-transposable element functions in Drosophila. Cell 38: 135-146.

Lindsley, D.L. and E.H. Grell. 1968. Genetic variation of Drosophila melanogaster. Carnegie Institution of Washington, Publ. No. 627.

Maniatis, T., E.F. Fritsch, and J. Sambrook. 1982. Molecular cloning: A laboratory manual. Cold Spring Harbor Laboratory, Cold Spring Harbor, New York.

Padgett, R.W., D. St. Johnston, and W.M. Gelbart. 1987. A transcript from a Drosophila pattern gene predicts a protein homologous to the transforming growth factor $-\beta$ family. $\mathrm{Na}$ ture 325: 81-84.

Pardue, M.L. and J.C. Gall. 1975. Nucleic acid hybridization to the DNA of cytological preparations. Methods Cell Biol. 10: $1-16$.

Rubin, G.M. and A.C. Spradling. 1982. Genetic transformation of Drosophila with transposable element vectors. Science 218: $348-353$.

Segal, D. and W.M. Gelbart. 1985. Shortvein, a new component of the decapentaplegic gene complex in Drosophila melanogaster. Genetics 109: 119-143.

Spencer, F.A., F.M. Hoffmann, and W.M. Gelbart. 1982. Decapentaplegic: A gene complex affecting morphogenesis in Drosophila melanogaster. Cell 28: 451-461.

Van der Meer, J.M. 1977. Optically clean and permanent whole mount preparations for phase-contrast microscopy of cuticular structures of insect larvae. Dros. Inf. Serv. 52: 160.

Vigue, C. and W. Sofer. 1976. Chemical selection of mutants that affect $a d h$ activity in Drosophila. III. Effects of ethanol. Biochem. Genet. 14: 127-135. 


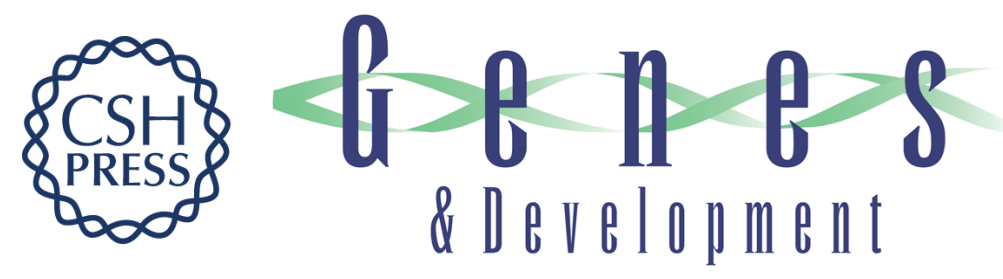

\section{Identification in transgenic animals of the Drosophila decapentaplegic sequences required for embryonic dorsal pattern formation.}

F M Hoffmann and W Goodman

Genes Dev. 1987, 1:

Access the most recent version at doi:10.1101/gad.1.6.615

References This article cites 15 articles, 4 of which can be accessed free at: http://genesdev.cshlp.org/content/1/6/615.full.html\#ref-list-1

License

Email Alerting

Receive free email alerts when new articles cite this article - sign up in the box at the top Service right corner of the article or click here.

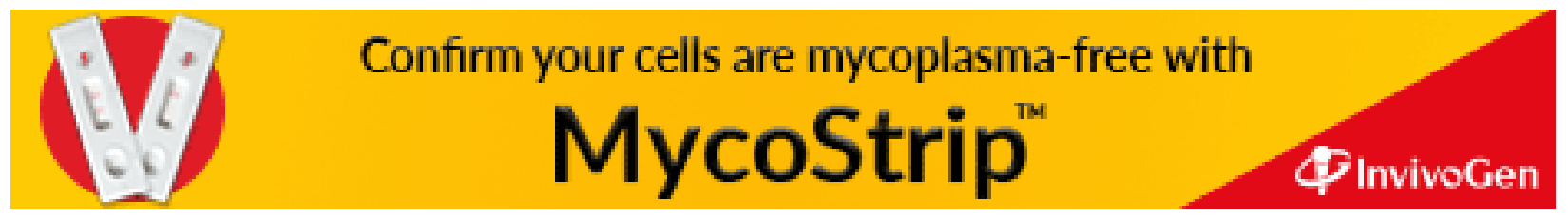

